\title{
Naive Bayesian decision model for the interoperability of heterogeneous systems in an intelligent building environment
}

\begin{abstract}
The environment of an intelligent building includes systems and sensors with significant heterogeneity. With the growth of heterogeneous devices and sensor technologies in intelligent buildings, designers are often faced with more interoperation complexities among heterogeneous systems that are data-intensive in nature. Conversely, performing decision making on these systems with a variety of building data is not efficient in terms of adapting to a changing environment. Current interoperation solutions for intelligent buildings focus on drycontact and static rule-based systems to solve interoperability in intelligent buildings. Although static rule-based systems are efficient, the bottleneck can be in the area of the scalability of heterogeneous systems that will affect the efficient performance of interoperations. Therefore, to ensure a timely decision-making mechanism among heterogeneous systems, the performance delay and interpretation capability of systems should be improved. This paper offers a framework that improves the communication responses and the ability of automated decision support among the heterogeneous systems with a rule-based repository for the management of intelligent buildings. The implementation of the framework was performed with several systems to show their adaptation to joint interoperation. It is also interwoven with Web services to offer unified integration among heterogeneous systems. Testing of the framework was performed in a local area network (LAN) setting and proved to be reliable in solving interoperation problems for intelligent buildings.
\end{abstract}

Keyword: Intelligent buildings; Naive Bayesian; Decision-making 